\section{Lebenszeichen von gesetzgeberischen Datenschutzvorhaben}

\section{Beschäftigtendatenschutz}

Die SPD-Fraktion im Deutschen Bundestag hat am 27.09.2011 einen Antrag (BT-Drucksache 17/7176) zur Wiederaufnahme des Gesetzgebungsvorhabens gestellt.

Ausgangspunkt ist die bestehende Situation:

Im September 2010 hat die Bundesregierung einen Gesetzentwurf zur Regelung des Beschäftigtendatenschutzes vorgelegt (Bundestagsdrucksache 17/4230). Hiernach sollen Beschäftigtendaten durch Änderungen im Bundesdatenschutzgesetz geschützt werden.

Das Anliegen der Bundesregierung ist wichtig, seine Umsetzung aber unzureichend. Schon vor Vorlage des Gesetzentwurfs hatten Datenschützer und Arbeitsrechtler zu Recht gefordert, den Beschäftigtendatenschutz in einem eigenständigen Gesetz zu regeln. Fragen des Beschäftigtendatenschutzes müssen genau und rechtssicher beantwortet werden. Dafür reicht die Systematik des allgemeinen Datenschutzrechts des Bundesdatenschutzgesetzes nicht aus.

Diese Bedenken wurden in einer öffentlichen Anhörung im Innenausschuss des Deutschen Bundestages am 23. Mai 2011 untermauert. Die Ergebnisse der Anhörung machen deutlich, dass der von der Bundesregierung vorgelegte Gesetzentwurf ungeeignet ist, die Persönlichkeitsrechte der Beschäftigten effektiv zu schützen. Dies wurde durch mehrere Sachverständige bestätigt. Hinzu kommt, dass es nicht nur bei den im Gesetzentwurf der Bundesregierung getroffenen Regelungen bleiben soll. Vielmehr ergibt sich aus einem von den Berichterstattern der Koalitionsfraktionen der CDU/CSU und FDP vorgelegten Eckpunktepapier (Ausschussdrucksache 17(4)255), dass die Persönlichkeitsrechte der Beschäftigten noch weiter untergraben und der Datenschutz Beschäftigter dem Interesse von Arbeitgebern einseitig untergeordnet werden sollen.

Der Antrag fordert die Bundesregierung dazu auf den Beschäftigtendatenschutz in einem eigenständigen Gesetz zu regeln, das die Beschäftigten schützt sowie die Rechte der Betriebs- und Personalvertretungen entsprechend ausbaut.

- Das Gesetz soll die Datenerhebung, -verarbeitung und -nutzung ermöglichen, soweit dies für den ordnungsgemäßen Betrieb erforderlich ist. Dabei müssen jedoch die Privatsphäre der Beschäftigten geschützt und eine Wiederholung vorangegangener Datenschutzskandale verhindert werden.

- Es ist dafür Sorge zu tragen, dass gegenüber dem vorgelegten Gesetzentwurf keine Verschlechterungen zulasten von Beschäftigten aufgenommen werden.

Am 09.11.2011 wurde in einer Fragestunde im Deutschen Bundestag das Thema aufgerufen:

- Frage des Abgeordneten Dr. Konstantin von Notz (BÜNDNIS 90/DIE GRÜNEN) (Drucksache 17/7583, Frage 57): Wann wird die Bundesregierung dem Deutschen Bundestag die angekündigte Überarbeitung ihres Entwurfs eines Gesetzes zur Regelung des Beschäftigtendatenschutzes (Bundestagsdrucksache 17/4230) vorlegen?
- Antwort des Parl. Staatssekretärs Dr. Ole Schröder (BMI): Die Bundesregierung hat den Entwurf eines Gesetzes zur Regelung des Beschäftigtendatenschutzes am 25. August 2010 beschlossen. Mit der ersten Lesung des Gesetzentwurfs am 24. Februar 2011 hat das parlamentarische Gesetzgebungsverfahren begonnen. Es ist nunmehr Angelegenheit der Fraktionen des Deutschen Bundestages, für erforderlich gehaltene Änderungen des Gesetzentwurfs vorzunehmen.

\section{Stiftung Datenschutz}

Entgegen wiederholter Ankündigungen zur Einrichtung der Stiftung im Jahr 2011, ist - nach einem Bericht der Dresdner Neuesten Nachrichten vom 08.11.2011 - nun die Verschiebung des Starts in das Jahr 2012 mit weiterhin erforderlichen Abstimmungen zur Satzung begründet. Auch zur dauerhaften Finanzierung gibt es noch Unklarheiten.

Als bevorzugter Sitz der Stiftung ist Leipzig im Gespräch.

\section{Einheitliches Datenschutzrecht in Europa durch Verordnung?}

Das Datenschutzrecht soll in Europa vereinheitlicht werden, dazu plant die EU-Kommission das Rechtsinstrument der Verordnung, die unmittelbare Geltung für sämtliche Mitgliedstaaten entfaltet, dies verkündete Paul Nemitz, Direktor der Direktion Grundrecht und zuständig für das Datenschutzrecht, anlässlich des Eröffnungsplenums der 35. Datenschutzfachtagung (DAFTA) der Gesellschaft für Datenschutz und Datensicherheit (GDD) am 17.11.2011 in Köln.

Nach Darstellung von Nemitz besteht ein Bedürfnis, den Datenschutz durch eine Verordnung zu regeln, da hierdurch die Komplexität des Datenschutzrechts vermieden und eine EU-weite Vereinheitlichung verwirklicht werden. Man komme damit auch einem Wunsch der Wirtschaft nach, die sich einheitliche und verständliche Regelungen wünscht. Insbesondere kleine und mittelständische Unternehmen benötigen verständliche europaweit geltende Normen.

Die geplante EU-Verordnung soll auch die Zuständigkeit der Datenschutzaufsichtsbehörden konkretisieren. Sofern Unternehmen in mehreren Mitgliedstaaten tätig sind, soll diejenige Aufsichtsbehörde zuständig sein, bei der sich der Hauptsitz des Unternehmens befindet („One-Stop-Shop“). Dem Bürger hingegen soll allerdings die Möglichkeit verbleiben, seine Datenschutzrechte bei der für ihn zuständigen Aufsichtsbehörde geltend zu machen. Die Sanktionsmöglichkeiten der Aufsichtsbehörden sollen erheblich verstärkt und effektiver ausgestaltet werden.

Das Prinzip der Selbstkontrolle durch betriebliche Datenschutzbeauftragte soll europaweit vereinheitlicht werden. Die EU-Kommission plant eine verpflichtende Bestellung von betrieblichen Datenschutzbeauftragten bei Unternehmen, die die Größe eines kleinen oder mittelständischen Unternehmens überschreitet. Zugleich sollen diese Unternehmen bei ihrem IT-Einsatz auf eine Folgenabschätzung im Datenschutz verpflichtet werden. Ein entschei- 